$$
R\left(j, \varphi_{p}\right)=\sum_{L} S_{i k} \cdot l_{i k}\left(j, \varphi_{p}\right)
$$

where the summation along the direction $L$ includes all disk pixels of the object $(i, k)$ that graze the projection of pixel $\left(i, \varphi_{p}\right)$ of the image $(i, k=-4,+4 ; j=-7 .+7) ; S_{i k}$ is the absorption coefficient in pixel $(i, k) ; l_{i k}$ is the effective length of the absorption segment mentioned above:

$$
l_{i k}\left(j, \varphi_{p}\right)=\left\{\begin{array}{l}
0, \text { for } l \equiv\left|i \cdot \cos \varphi_{p}+k \cdot \sin \varphi_{p}-j\right| \geq 1 \\
\frac{\left[2 \cdot \cos ^{-1}(2 d-1)-\sin \left(2 \cdot \cos ^{-1}\right)(2 d-1)\right]}{8(1-d)}, \text { for } d<1
\end{array}\right.
$$

As an example, Fig. $2 \mathrm{c}$ shows some intensity distributions projected at different angles that together form a "image," on the basis of which the density distribution in the object is reconstructed. The results of such reconstruction for 125 uniformly distributed rotation angles $\varphi_{p}$ are given in Figs. $2 \mathrm{~d}$ and $2 \mathrm{e}$.

Obviously, the number of pixels, projections, and intensity of the incident radiation must be matched in each individual case to the required accuracy of reconstruction and the spatial resolution. Important here is the fact that for given values of the accuracy and resolution the "limiting" properties of the SPIPR approach described earlier ensure the minimum possible radiation dose. This circumstance can also be characterized as follows: For a fixed radiation flux, SPIPR makes it possible to achieve the highest spatial resolution and the maximum reconstruction accuracy.

\title{
6. CONCLUDING REMARKS
}

We note once more that the above analysis does not exhaust the possibilities of the SPIPR in the fields of application we have mentioned; our aim was merely to explain the basic problems that arise in such a study. Particular attention is to be devoted to adequate allowance for a priori information, since the quality of reconstruction depends strongly on it. These questions will be considered in following publications.

We thank V. B. Biryukov for a helpful discussion.

\section{REFERENCES}

1. V. Yu. Terebizh, Astrofizika, 32, 327 (1990).

2. V. Yu. Terebizh, Astron. Astrophys. Transac., 1, No. 1 (1991).

3. V. Yu. Terebizh, Proc. of the Third ESO/ST-ECF Data Analysis Workshop, Garching, 22-23 April (1991),

4. V. Yu. Terebizh, Exp. Astronomy (in press).

5. V. Yu. Terebizh, O. K. Cherbunina, Yu. G. Cherbunin, and V. V. Biryukov, Astrofizika, 34, 91 (1991).

6. V. Yu. Terebizh and V. V. Biryukov, Astrofizika, 34, 233 (1991).

7. S. M. Rytov, Yu. A. Kravtsov, and V. I. Tatarskii, Introduction to Statistical Radiophysics, Pt. 2 [in Russian], Nauka, Moscow (1978).

8. D. L. Fried, J. Opt. Soc. Am., 55, 1427 (1965).

9. F. Roddier, Progress in Optics, Vol. 19 (ed. E. Wolf), North-Holland, Amsterdam (1981), p. 281.

10. A. Labeyrie, Astron. Astrophys., 6, 85 (1970).

11. A. Labeyrie, Progress in Optics, Vol. 14 (ed, E. Wolf), North-Holland, Amsterdam (1976), p. 49.

12. J. M. Beckers and L. E. Goad, Instrumentation for Ground-Based Optical Astronomy. Proc. of the Ninth Santa Cruz Summer Workshop (ed. L. B. Robinson), Springer, New York (1988), p. 315.

13. A. K. Jain, Fundamentals of Digital Image Processing, Prentice Hall, Englewood Cliffs (1989).

\section{CORRECTION}

In the paper V. Yu. Terebizh "Maximum likelihood image restoration. IV. Limiting resolution for given alternative" (Astrofizika, 33, No. 3, 409-427) the author made an error in one of the examples illustrating the general relations. Namely, in the expression (35) the operation of squaring the moduli of the spectral densities was omitted. The correct form of the expressions (35), (36), (38), and (39) is

$$
|\Delta S(f)|^{2}=4 \sin ^{2}(\pi g f)\left|S_{0}(f)\right|^{2},
$$




$$
\begin{gathered}
\theta=2\left[\left.\int_{-f_{R}}^{f_{R}}|T(f)|^{2} \cdot\left|S_{0}(f)\right| F\right|^{2} \cdot \sin ^{2}(\pi / f) d|| f_{\mathrm{R}}\right]^{1 / 2}, \\
r \cong K \cdot \frac{z_{a}+z_{B}}{\Psi}, \Psi \gg 1, \\
K=\frac{1}{2 \pi}\left[\int_{-f_{R}}^{f_{R}}\left|T(f)^{2} \cdot\right| S_{0}(f)\left(\left.F\right|^{2} \cdot\left(f ! f_{R}\right)^{2} d\left(f / f_{R}\right)\right]^{-1 / 2} .\right.
\end{gathered}
$$

Thus, for relatively bright objects in the approximation of deterministic image formation the smallest detectable displacement is inversely proportional to the first power of the signal-to-noise ratio, in contrast to the case then considered of double sources, in which the limiting separation of the components is inversely proportional to the square root of the signal-tonoise ratio. 